\title{
Clotting factor VIII cloned
}

\section{from George G. Brownlee and Charles Rizza}

ONE of the most exciting and ambitious goals of the biotechnology industry has been achieved. In papers that begin on page 326 of this issue, both Genentech, assisted by Tuddenham and colleagues of the Royal Free Hospital, London, and Genetics Institute, assisted by Fass and Knutson of the Mayo Clinic, Rochester, report the cloning of DNA for human blood clotting factor VIII:C and the production of the factor from cloned DNA expressed in mammalian cell lines.

Clotting factor VIII:C is the name given to the protein that is absent or defective in patients with classical haemophilia (haemophilia A), a sex-linked bleeding disorder of about 20 per 100,000 males. In the blood stream, factor VIII:C is associated with another protein, known as von Willebrand protein or factor VIII-related antigen, but factor VIII:C is solely responsible for the clotting activity of the complex (which is loosely termed factor VIII). Injection of factor VIII obtained from donor blood allows haemophiliacs to be successfully managed. But, unfortunately, the factor VIII preparations in use at the moment carry a high risk of transmitting bloodborne viruses such as non- $A$, non-B hepatitis virus. The virus that is thought to cause AIDS may also contaminate preparations and, although relatively few haemophiliacs have been affected so far, the fear of the disease has caused great anxiety to patients, their families and their doctors. A genetically-engineered factor VIII:C derived from a source other than blood should be free of such viruses. It should also be cheaper.

Both groups have used similar methods for the DNA cloning and expression of factor VIII:C and report very similar results. Oligonucleotide probes were synthesized on the basis of small sections of the amino acid sequence of purified factor VIII:C (either pig or human in origin), and used to screen an appropriate library of genomic clones to identify part of the factor VIII:C gene. The gene itself turned out to be extraordinarily long (180 kilobases), to have 26 exons and to occupy an estimated 0.1 per cent of the $X$ chromosome (see Gitschier et al. on page 326 for further details).

For expression of protein it was necessary to isolate cDNA clones. These were obtained either from a human $\mathrm{T}$-cell hybridoma line (Genentech) or from human liver (Genetics Institute) and sequence analyses allowed the derivation of the entire 2,332 amino acid sequences of the mature protein. Both groups (but see Vehar et al. on page $\mathbf{3 3 7}$ for an extended discussion), note that the protein is highly glycosylated and has an obvious domain structure with predictable homology to another clotting factor, factor $\mathrm{V}$, but also an unpredicted homology to ceruloplasmin - a serum protein believed to be involved in copper ion transport in the blood. The protein sequence also allows the definition of the main sites that are cleaved by thrombin in the process of activation of factor VIII:C.

The expression of factor VIII:C in mammalian cells required the reconstruction of a full length cDNA clone and its attachment to a viral promoter sequence. When this construction was introduced into either a hamster kidney cell line (Genentech) or a monkey kidney cell line (Genetics Institute), a human factor VIII:C-like activity was secreted into the media in which the cells were grown. The concentrations of factor VIII:C-like activity in the media were about one per cent (Genetics Institute) or seven per cent (Genentech) of the normal plasma concentration as assayed by a sensitive biochemical test based on the activation of factor $\mathrm{X}$ and hydrolysis of a chromogenic substrate. Furthermore, both groups showed that the secreted activity (further purified by affinity chromatography at Genentech) was able to correct the clotting time of plasma from a haemophiliac. Evidence that these assays are indeed a measure of factor VIII:C and not of some other clotting factor or non-specific activity, was provided by two additional tests carried out by both groups. In the first, the clotting activity was shown to be increased by thrombin, a normal physiological activator of factor VIII:C. In the second, it was shown to be inhibited by specific factor VIII:C antibodies. The Genentech group also showed that, like authentic factor VIII:C, their substance bound reversibly to the von Willebrand protein immobilized on an affinity column and that its activity was destroyed by protein $\mathrm{C}-$ a serine protease of plasma that can inactivate factor VIII:C.

What is the outlook for treatment of haemophiliacs? It is clear from previous experience with genetically-engineered products that we must anticipate some delay, perhaps 3-5 years, before a safe, effective and clinically-tested product is on the market. The expression of factor VIII:C reported by both groups is very low and it is probable that more effective vectors or host-cell systems will have to be devised. Effective large-scale tissue culture and purification methods will also have to be developed. These may differ from existing procedures for purification of factor VIII from blood. We emphasize the need for a high purity product to guard against the possibility of any pathogenic material from the culture being introduced into haemophiliacs. It would be unacceptable to eliminate the risk of AIDS but substitute a new hazard. There remains the possibility that the genetically-engineered factor VIII:C, although of proven activity in a test-tube assay, may not be as effective in vivo. This can only be adequately tested by clinical trials of the product, the outcome of which must be eagerly awaited.

George G. Brownlee is in the Sir William Dunn School of Pathology, South Parks Road, Oxford, OXI $3 R E$ and Charles Rizza is director of the Oxford Haemophilia Centre, Churchill Hospital, Oxford OX3 7JL, UK.

\section{Astrophysics}

\section{Evolution of star-planet systems}

\section{from Geoffrey Bath}

A PROBLEM at the heart of stellar astrophysics is the role of angular momentum in the evolution of stars. This statement may seem controversial, given the profound success of stellar evolution theory in describing quantitatively the nucleosynthesis of the elements and the evolutionary transformation in the structure of stars as they perform their function as cosmic alchemists. The theory of stellar evolution has been largely developed without involving rotation in any major way. But a recent paper by $\mathbf{M}$. Livio and N. Soker (Mon. Not. R. astr. Soc. 208, 763; 1984) raises the dramatic possibility that the evolutionary fate of many single stars accompanied by planets could be both binary star systems and exploding variables.

Certainly, in the case of the Sun, present angular momentum, if rotation is uniform, is quite unimportant in determining structure or immediate evolutionary future. The Sun cannot, however, be considered as an isolated unit; it is only one part - albeit by far the most massive part - of the Solar System. The angular momentum of the planets is sixty times greater than that of the Sun's own, assuming solid-body rotation in the inner core. Furthermore, the initial solar nebula, with a minimum mass of some 3 per cent of the Sun's mass, must have possessed an even greater fraction of angular momentum.

In the case of binary stars, angular momentum plays a very important part in determining their present binary state and subsequent evolution. In binaries, angular momentum decreases with decreasing separation. Even for W Ursae Majoris 